\title{
Research on KAAS-based Social Knowledge Collaboration Service Mechanism and Application
}

\author{
Yuan Rao, \\ Lab of Social Intelligent and Complex Data Process, \\ School of Software, Xi'an Jiaotong University, \\ No. 28, Xianning West Road, 710049 \\ Xi'an, Shannxi Province, China \\ e-mail: raoyuan@mail.xjtu.edu.cn
}

\author{
Shaochuan Chen \\ Lab of Social Intelligent and Complex Data Process, \\ School of Management, Xi'an Jiaotong University, \\ No. 28, Xianning West Road, 710049 \\ Xi'an, Shannxi Province, China \\ e-mail: sccheng@mail.xjtu.edu.cn
}

\begin{abstract}
Knowledge as a Service (KAAS), which merges the whole architecture of knowledge into service process, is proposed and defined in the paper. Based on the BNF definition of KAAS, the new knowledge service mechanism is provided to aggregate the knowledge resources together for satisfying various users' personalized requirements. In addition, a social knowledge collaboration service algorithm and strategy for knowledge classification are proposed, which optimizes and aggregates all these knowledge resources by two core engines, such as knowledge matching engine and requirement matching engine. Furthermore, the personalized knowledge recommendation and social collaboration service mechanisms are studied in the paper to optimize the knowledge service capability and process. Then, a KAASbased platform with social knowledge service patterns, named EKNOWARE, is developed under the social computing and knowledge collaboration service environment.
\end{abstract}

Keywords- Collaboration Service; KAAS; Social Knowledge Management; Knowledge Mining;

\section{INTRODUCTION}

At present, knowledge as a service (KAAS) is becoming next trend and new pattern to organize the data, information and knowledge to provide a solution for users' personalized knowledge requirements in big data era[1,2]. Due to adopting the web 2.0 technology and many web mining algorithms, KAAS not only changes the mechanism about knowledge management in social network environment for collaborative innovation among the users in COP, but also accelerates the research into the intelligent knowledge aggregation and knowledge graph service [3]. In addition, according to the principles of service science, KAAS service is to allow users "pay-per-use" and access to "specialized" knowledge on demand. Eng K. Chew [4] provided a methodology about KAAS classification scheme based on the business model principles. Therefore, the mechanism and metrics about KAAS are investigated in the paper to utilize various knowledge resources under the social network environment.

The rest of this paper is organized as follows: the definition of KAAS, a model of Knowledge as a Service, is provided based on the comparing between knowledge service and knowledge management. Section 3 and section 4 provide a knowledge collaboration service mechanism based on the KAAS and investigate an optimized algorithm to build the architecture for knowledge service platform. A KAAS-based collaborative service platform, named EKNOWARE, is illustrated in the Section 5 with multielements under the social network. Finally, the conclusion and future research works is presented in Section 6.

\section{THE DEFINITION ABOUT KAAS}

Many commercial KM software have been introduced various functions to different users with personalized knowledge storage and application [5]. All these software can excavate out the potentiality of reusing existed knowledge resources to the maximum extension and promote the knowledge reused level incretion in special domain. Whereas it is obviously insufficient to provide knowledge resources for reused to solve cross-domain problem. In particularly, it is important how to acquire the content of knowledge and integrate these knowledge into a solution to form new knowledge products and services. Therefore, Knowledge as a Service (KaaS) is an on-demand, whereneeded approach [6] to knowledge acquisition, which integrates the knowledge and service together and turns the knowledge resources into products and service for valueadded.

Define 1: Knowledge as a Service (KAAS) Model means that the knowledge can be organized and delivered to different users as service to satisfy their own personalized requirement. The formally definition about KAAS can be described by BNF style in figure1.

Wherein, knowledge resources is a finite set, denoted as $\mathfrak{R}=\left\{\mathfrak{R}_{i} \mid 0<i<n\right\}$, and each knowledge resource $\mathfrak{R}_{i}$ can also be composed of a finite set with vectors of knowledge features in various knowledge classification $\mathfrak{R}_{i}=\left\{\kappa_{i, j} \mid C_{k}, 0<j<m, 0<k<n\right\}$. The features vector can be analyzed and aggregated together to form a unique knowledge description under the constrain of $C_{k}$.

Knowledge requirement also is a finite set, denoted as $\rho=\left\{\rho_{l} \mid 0<l<n\right\}$. Where, $\rho_{l}$, as a knowledge requirement element, can be defined by another two subtuple: $\rho_{l}=<R P_{l}, R C_{l}>$. In this tuple, $R P_{l}$ denotes the 
requirement specification with some elements, such as requirement tag, content, feature vector, etc. $R C_{l}$ can be denoted as requirement constrains with user's constrain, behavior constrain and relative knowledge constrain, which can be formerly described as $R C_{l}=<U P_{l}, B P_{l}, C P_{l}>$. In this sub-tuple, $U P_{l}$ denotes user's knowledge profile model with $U P_{l}=\{$ User's Tags $\mid$ User's knowledge characteristics $\mid$ User's knowledge favorites $\mid\}$, which focuses on the different logical hierarchy for user's profile characteristics with tags, user's statutes characteristics. $B P_{l}$ integrates the user's behaviors and operations into a correlation knowledge vector space with $B P_{l}=\left\{w_{k} e_{l, k} \mid 0<l, k<n\right\} \quad$, where, $w_{k}$ means the weight for $e_{l, k}$, which is a finite set about $l t h$ user to operate kth knowledge object with association behavior characteristics as $e_{l, k}=\left\{l k_{i} \mid 0<i<n\right\}$. Then, $C P_{l}$ denotes a finite set of lth user's knowledge requirement with $C P_{l}=\left\{r_{l, h} \mid 0<l, h<n\right\}$. Based on the existed requirement characteristics, user can acquire and filter new knowledge solution by these constraint conditions, such as user's knowledge profile model, the knowledge and requirement vector model for individual. Therefore, how to analyze the similarity of knowledge contents under these tight constrains condition is a critical mechanism about knowledge extracting and filtering.

Knowledge Service Mechanism is denoted as $\phi$ with $\phi=\left\{S_{l, \rho} \mid A_{k}, 0<k<n\right\}$, where, $S_{l, \rho}$ means that a knowledge solution to adopt algorithm $A_{k}$ for satisfying the lth user's knowledge requirement $\rho_{l}$. The solution $S_{l, \rho}$, composed by a serial of similar knowledge resources and users' requirements, is varied with the change of algorithms and provided the different knowledge service level (KSL) [7]. Therefore, the knowledge as a service should be delivered

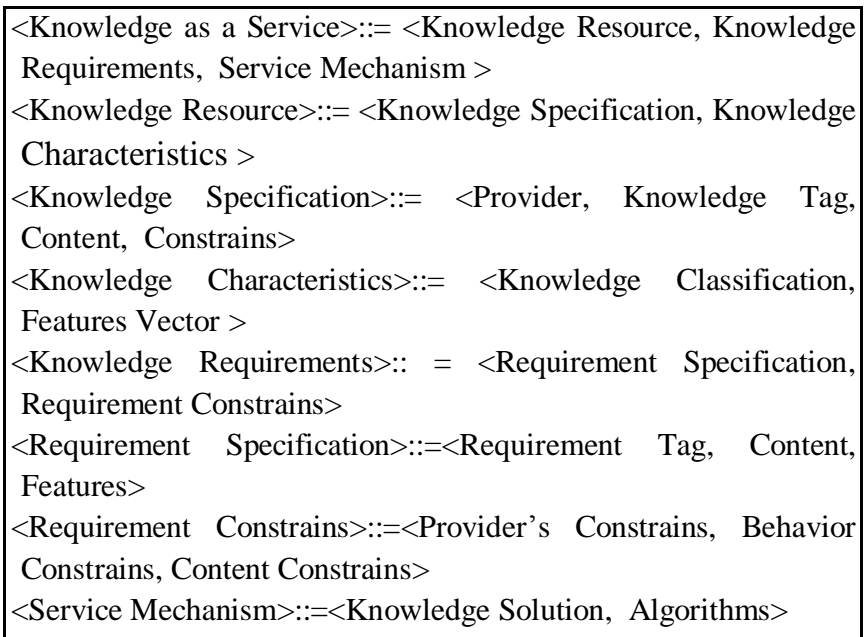

$<$ Service Mechanism $>::=\langle$ Knowledge Solution, Algorithms $>$

Figure 1. The BNF definition about Knowledge as a Service the knowledge solution with various algorithms to satisfy the different user's knowledge requirements.

\section{Social KNOWLEDGe COLlaboration SERVICE MECHANISM}

The social knowledge collaboration is a process from a personalized knowledge creative to exchange and recommendation among the multi-member within the web community group (COP), which needs a dynamic mechanism for knowledge query, transfer and share with multi-user collaboration. In addition, in order to enhance the efficiency about knowledge resources sharing, the strategy of knowledge recommendation service should be provided for knowledge combination, which also can merge the different user's favorites into knowledge consumption with current user's favorite.

In addition, because the knowledge resources are decomposed into many different types in KAAS meta-model, the similarity of various knowledge resources needs to be reckoned and aggregated by knowledge feature vector under knowledge requirement specification $R P_{l}$. At the same time, with the constantly increasing about user access and operation in social network website, the information about user's favorites, tags characteristic and knowledge exchange between group members are enlarged rapidly. Because the user's behavior characteristics have been merged into knowledge document vector, the similarity degree, between user's profile and knowledge resources, can be calculated for knowledge matching and collaborating by the formula above with knowledge recommendation service.

Therefore, a dynamic unified characteristic model for knowledge document should be established in the process of indexing knowledge document with collaboration filter. Moreover, the user's behavior profile ${ }^{B P_{l}}$ and knowledge requirement $C P_{l}$ should be integrated into the unified characteristic model. This unified model should be avoid of the problem about "cool data" when the data of scoring matrix is deficiency. Furthermore, a unified social knowledge collaborative service mechanism, which optimizes the TFIDF and CDF algorithms for knowledge classify, is illustrated as follow:

Step1: (Data Preparation)

//To define the profile model about knowledge document

$$
\begin{gathered}
\mathrm{D}_{\mathrm{fp}}=\left\{\mathrm{D}_{\text {title }}, D_{\text {tag }}, D_{\text {content }}\right\}, \text { where, } \\
\mathrm{D}_{\text {title }}=\left\{\left(\text { Term }_{i}^{\text {title }}, W_{i}\right) \mid 0<i<n\right\}, \\
\mathrm{D}_{\mathrm{tag}}=\left\{\left(\text { Term }_{i}^{\text {tag }}, W_{i}\right) \mid 0<i<n\right\}, \\
\mathrm{D}_{\text {content }}=\left\{\left(\text { Term }_{i}^{\text {content }}, W_{i}\right) \mid 0<i<n\right\}
\end{gathered}
$$

//to define the user's profile model, the different type of user with different weight

$$
\begin{gathered}
\mathrm{U}_{\mathrm{fp}}=\left\{\mathrm{U}_{\text {profile }}, U_{\text {tag }}\right\}, \text { where } \\
\mathrm{U}_{\text {profile }}=\left\{\left(\text { Term }_{i}^{\text {profile }}, W_{i}\right) \mid 0<i<n\right\}, \\
\mathrm{U}_{\mathrm{tag}}=\left\{\left(\text { Term }_{i}^{\text {tag }}, W_{i}\right) \mid 0<i<n\right\}
\end{gathered}
$$


Step2: ( the Weight of Term Frequency for Knowledge Document)

//based on the category of document, the total weight of term $_{j}$ in ith document as follow:

$$
\varpi_{i j}=\varpi_{i j}^{k r}+\varpi_{i j}^{u_{-} \text {creater }}+\varpi_{i j}^{u_{-} \text {requiree }} \text {, and normalization }
$$

processing as:

$$
\varpi_{i j}=\frac{w_{i j}}{\sqrt{\sum_{l}\left(w_{l j}\right)^{2}}}, 0<l \leq n, 0<i \leq n
$$

Where, $\varpi_{i j}^{k r} 、 \varpi_{i j}^{u+\text { creater }}$ and $\varpi_{i j}^{u \_ \text {requiree }}$ demote that the weight of $t_{\text {erm }}$ exists in ith knowledge document, the creator's characteristics vector, and the user's requirements, respectively. The value of whole weight is calculated with TF-IDF algorithm as follow:

$$
\begin{aligned}
& \varpi_{i j}^{k r}=T F_{i j}^{k r} * I D F_{j}^{k r} \\
& =\left(\frac{\text { term }_{j}^{\text {title }}}{D^{\text {title }}} W^{\text {title }}+\frac{\text { term }_{j}^{\text {tag }}}{D^{\text {tag }}} W^{\text {tag }}+\frac{\text { term }_{j}^{\text {content }}}{D^{\text {content }}}\right) * \log _{2}\left(\frac{N}{d f_{j}^{D_{f p}}}\right) \\
& \varpi_{i j}^{u_{-} \text {creater }}=\left(\frac{\text { term }_{j}^{\text {profile }}}{U^{\text {profile }}} W^{\text {profile }}+\frac{\text { term }_{j}^{\text {tag }}}{U^{\text {tag }}} W^{\text {tag }}\right) \\
& \varpi_{i j}^{u+\text { require }}=\left(\frac{\text { term }_{j}^{\text {profile }}}{U^{\text {profile }}} W^{\text {profile }}+\frac{\text { term }_{j}^{\text {tag }}}{U^{\text {tag }}} W^{\text {tag }}\right)^{*} \log _{2}\left(\frac{M}{d f_{j}^{U}}\right)
\end{aligned}
$$

Step3: ( the Measure of Cosine Similarity between Knowledge Documents )

$$
\begin{aligned}
& \forall \operatorname{Doc}_{i}, \operatorname{Doc}_{k}, i \neq l, 0<i, k<n \\
& \operatorname{CosSim}\left(\operatorname{Doc}_{i}, \operatorname{Doc}_{k}\right)=\frac{\sum_{i=1}^{n} \sum_{k=1}^{m}\left(w_{i j} \bullet w_{k j}\right)}{\sqrt{\sum_{i=1}^{n} w_{i j}{ }^{2} \bullet \sum_{k i=1}^{m} w_{k j}{ }^{2}}}
\end{aligned}
$$

Step4: (Dynamic Sorting Document by Similarity)

// sorting the document by similarity

$$
\text { Sort }\left(\operatorname{Cos} \operatorname{Sim}\left(\operatorname{Doc}_{i}, \operatorname{Doc}_{k}\right)\right)
$$

Step5: (Dynamic Satisfaction Degree between Knowledge Document and requirement)

// The calculation of satisfaction degree between user's requirement and knowledge documents

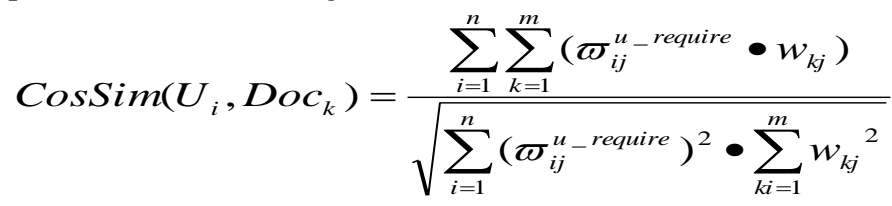

Because the user's behavior characteristics have been merged into knowledge document vector, the similarity degree, between user's profile and knowledge resources, can be calculated for knowledge matching and collaborating with knowledge recommendation service. Then,

$\operatorname{sort}\left(\operatorname{CosSim}\left(U_{i}, \operatorname{Doc}_{k}\right)\right) / /$ sorting by requirement

Step6: (knowledge collaboration service )

//clustering the relative knowledge by user's requirement $U_{i}$

$$
\text { Cluster(topN } \left.\left(\operatorname{Doc}_{i} \mid \operatorname{Doc}_{k}\right)\right), \quad 0<i<n
$$

In this step, we mix up the step5 with step4 to list top $\mathrm{N}$ knowledge documents, which have higher similarity degree to user's knowledge requirement $U_{i}$. And then, put these knowledge documents together to cluster and acquire the knowledge solution service with knowledge aggregation for different demanders.

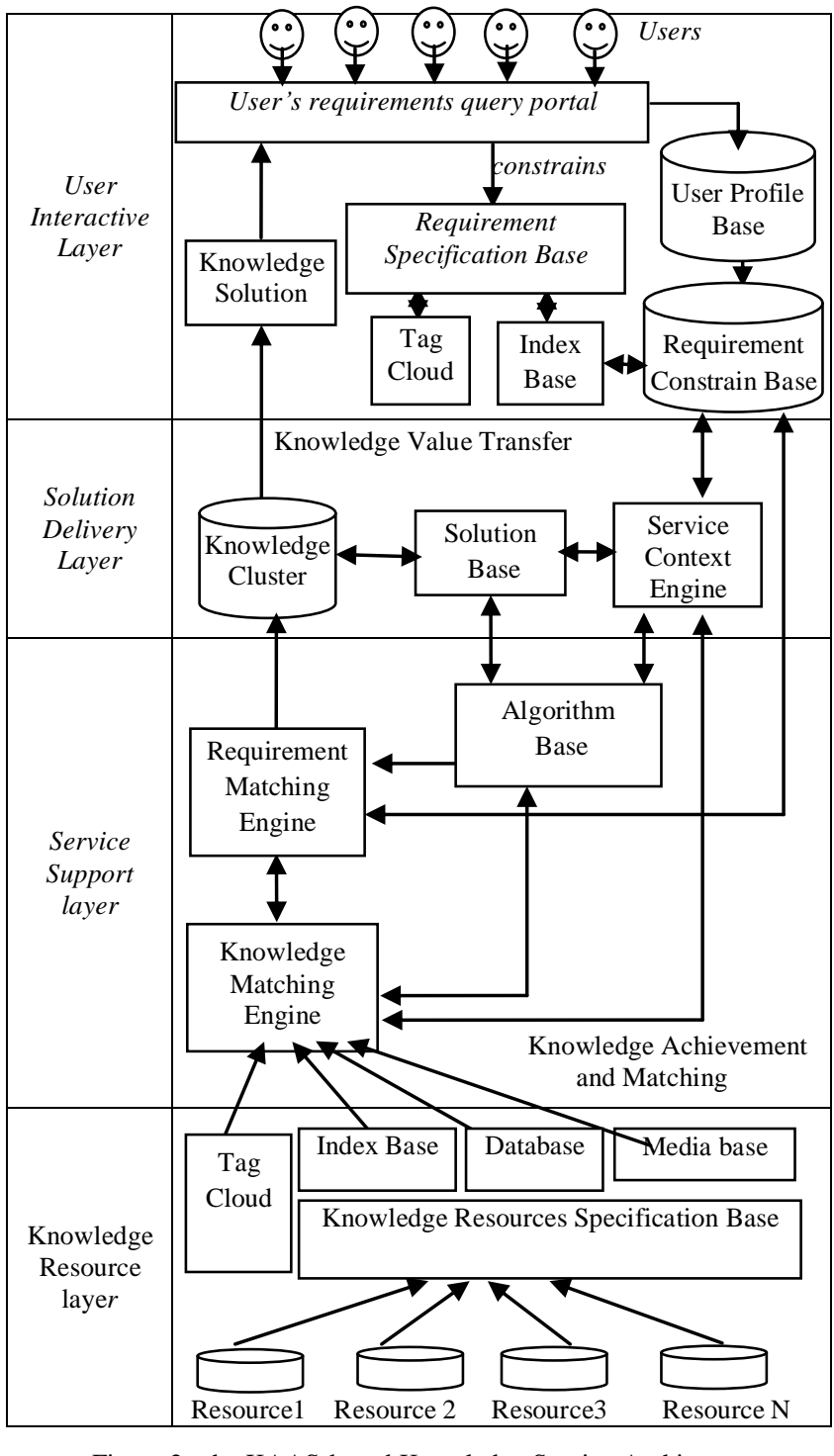

Figure 2. the KAAS-based Knowledge Service Architecture 


\section{THE KAAS-BASED KNOWLEDGE SERVICE ARCHITECTURE}

Based on the above-mentioned definition of KAAS and the social knowledge collaboration service mechanism, KaaS-based knowledge service can realize the knowledge availability by analyzed data, information and knowledge value-added, which also outputs some advices, answers or facilitations with knowledge clustering to meet external user's knowledge requirements. Therefore, knowledge service is a kind of social network-based intelligence with self-service and on-demand service with four service layers, shown in figure 2.

The first layer is composed of various knowledge resources, which can be cleared and normalized by knowledge specification. The knowledge resources specification base, which built by index base, database, media base and knowledge tags, is a basis of knowledge analysis and aggregation. In addition, the knowledge tags can put the different knowledge with same tags into one classification contains for aggregating the knowledge together.

The second layer is service support layer, which puts various data resources into Knowledge Matching Engine and executes the step3 and step4 to acquire the similar knowledge documents in above-mentioned algorithm. This engine can match the right knowledge documents from knowledge resources specification base to aggregate the high similarity knowledge documents together. Then, based on the user's requirements query, user's profile model, requirement specification base and requirement constrain base from the fourth layer, we can calculate the similarity degree between user requirement feature vector and knowledge feature vector into the Requirement Matching Engine with step5 in above algorithm in section III.

The third layer is solution delivery layer, which clusters the various related knowledge, filtered by Requirement Matching Engine and Knowledge Matching Engine, to form the knowledge service solution. These solution stores into the Solution Base in one hand, and provides to different users by knowledge cluster in another hand.

Then, all these solution can pull the new knowledge to users by KAAS-based service with different knowledge client in the fourth layer, i.e. the user interactive layer, which provides an interactive portal between user and knowledge service engine. In addition, the user's knowledge query and knowledge profile model should be build in this layer, which provides a fundamental structural information and a serious of constrain mechanisms.

All these four layers in knowledge services architecture provide an access portal for the non-specialist users to acquire the knowledge service by unstructured and scattered information aggregation in social knowledge sharing. Then, how to provide right knowledge service application to different users under the context environment are important issues. In this paper, a platform of knowledge acquisition and services, named EKNOWARE, is build, which using social knowledge network as a backbone. Based on the special design about community knowledge sharing and personal knowledge profile model, the system ensures that knowledge service could be improved the user potential business in knowledge consumption.

\section{KNOWLEDGE SERVICE PLATFORM AND APPLICATION}

Based on the above-mentioned service mechanism and system architecture, a social knowledge collaboration service platform, named Eknoware (http://eknoware.com), is build in campus network environment in China. The knowledge requirement specification, user's profile model and sharing mechanism in this platform are introduced in the social network environment. Today, more than 200,000 users, from more 40+ universities or other organization in china, have visited EKNOWARE Platform and provided many knowledge resources and some operation information, which could help us to analyze the user's behavior and knowledge sharing requirement.

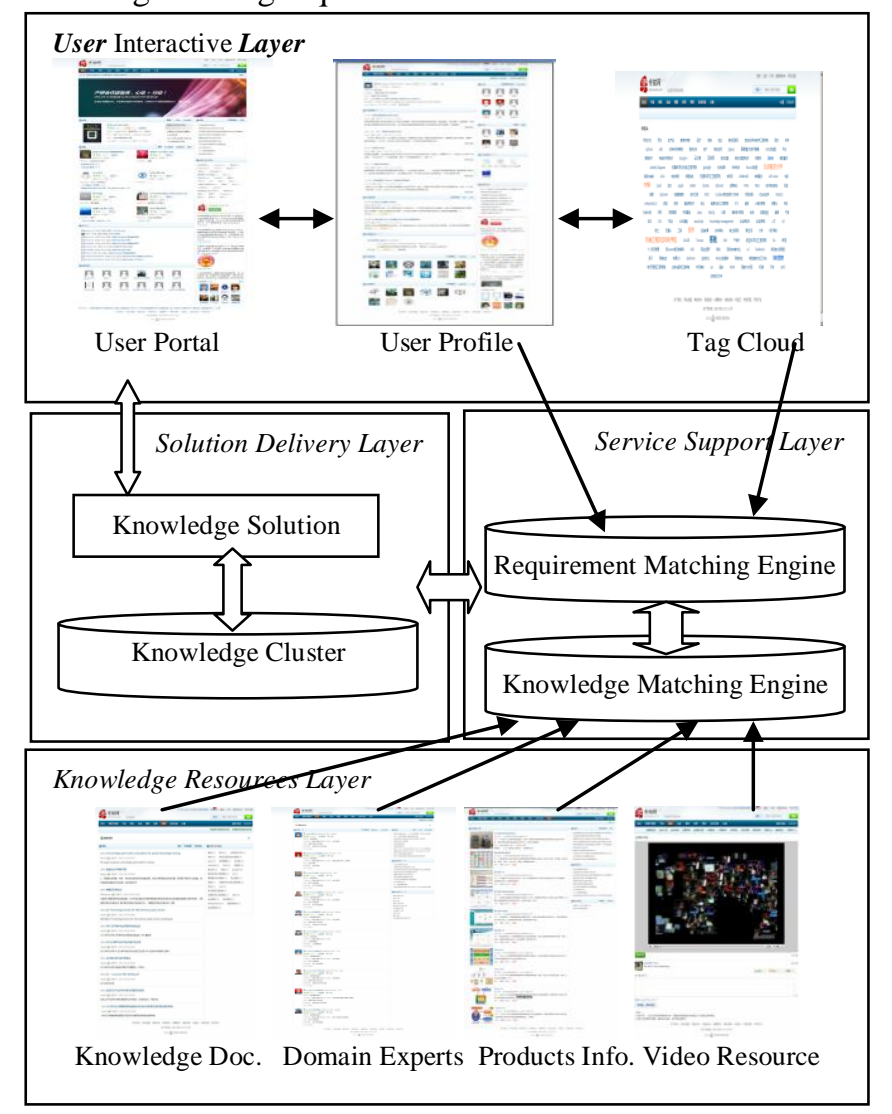

Figure 3. Eknoware, the KAAS-based Knowledge Service Platform (http://eknoware.com)

In addition, this social collaboration service platform provides two core engines, i.e. knowledge Matching Engine and Requirement Matching Engine, to analyze and cluster the knowledge resources for various users requirement. At same time, these knowledge resources and data come from the users in the platform to finish their operation, such as users group community, user profile space, and patent resources and so on, to share and aggregate these knowledge resources. In specifically, a personal knowledgebase is provided which can not only exchange the knowledge 
between different persons, but share the knowledge between different knowledge groups. So, the knowledge contents become a flow from one person to others person with new knowledge with value-added. The architecture about knowledge service platform is illustrated in figure 3 .

\section{THE CONLUSTION AND FUTURE WORK}

Recently, many research works [8 10] focused on the KAAS and promoted the new "wave" to develop the knowledge service-based application in big data era. Gartner group survey [11] shows that the $70 \%$ knowledge come from the interpersonal communication in business organization, which promote social knowledge service mechanism will become an important trend for knowledge collaboration service. However, knowledge service reuses the knowledge resource and creates a new value-added service more efficiently.

Therefore, a BNF definition about KAAS is proposed in this paper firstly. And then, a social mechanism about knowledge collaboration Service with six steps is investigated under the algorithm about knowledge similarity degree. Furthermore, a KAAS collobration service Architecture is provided in cloud environment, which makes the knowledge available on a self-service, social networkbased collective intelligence and on-demand service with four knowledge service layers as follows: knowledge resource layer, service support layer, solution delivery layer and user interactive layer. All these four key layers for providing knowledge services are how to improve the access of unstructured and scattered information for the non-specialist users, and how to provide adequate information to knowledge workers. Furthermore, the algorithm and realization about these strategies are optimized and developed to build a knowledge service platform, named Eknoware, with a social knowledge service by personalized knowledge recommendation.

\section{ACKNOWLEDGMENT}

This paper is joint supported by "2012 National Torch Plan Project in China (2012GH571817)", "2012 the Fundamental Research Funds for the Central Universities in China(08143003)", "2012 Shanxi Province Key Scientific and Technological Project (2012K11-08, 2012K06-18)" and
“2012 XI'AN Science and Technology Project
(CX12178(3))".

\section{REFERENCES}

[1] R. Buyya, C.S. Yeo, S. Venugopa, J. Broberg, I. Brandic, "Cloud computing and emerging It platforms: Vision, hype, and reality for delivering computing as the 5th utility", Future Generation Computer Systems, Vol. 25 (6) (2009), pp. 599-616.

[2] Jeffrey Heer and Sean Kandel, Interactive analysis of big data, XRDS, Vol.19(1), 2012, pp.50 54, DOI: 10.1145/2331042.2331058

[3] Chaveevan Pechsiri, Rapepun Piriyakul, Explanation Knowledge Graph Construction Through Causality Extraction from Texts, Journal of Computer Science and Technology, September 2010 , Volume 25, Issue 5, pp 1055-1070.

[4] Eng K. Chew, Towards a methodology framework for designing a KAAS system, the proceeding of IEEE Computer Science and Service System (CSSS 2011), pp.2823 2826, DOI: 10.1109/CSSS.2011.5975059

[5] S. Liao, Knowledge management technologies and applicationsliterature review from 1995 to 2002, Expert Systems with Applications, Vol.25 (2) (2003), pp. 155-164.

[6] Ergazakis, K., Metaxiotis, K., and Psarras, I. Knowledge Management in Enterprises: A Research Agenda. Intelligent Systems in Accounting, Finance and Management, 2005.13 (1): p. 17-26.

[7] Hofmann, P \& Woods, D., Cloud Computing: The limits of Public Clouds for Business Applications, IEEE Internet Computing, 2010, vol. 14 , no. 6 ,p. $90-94$.

[8] Malcolm, D., The five defining characteristics of cloud computing [online], ZDNet, 2010, http://www.zdnet.com/news/the-five-definingcharacteristics-of-cloud-computing/287001.

[9] Cheong, KFR \& Tsui, The Roles and Values of Personal Knowledge Management: An exploratory study, VINE: The journal of information and knowledge management systems, 2010, vol. 40, no. 2, p. 204-27.

[10] Delic, K.A \& Riley, J.F., Enterprise Knowledge Clouds : Architecture and Technologies, Handbook of Cloud Computing, 2010, Springer, NY, p.239-254;

[11] S. Xu and W. Zhang, Knowledge as a service and knowledge breaching, in SCC 2005: Proceedings of the 2005 IEEE International Conferenceon Services Computing. Washington, DC, USA: IEEE Computer Society, 2005, p. 87-94. 